\title{
炭包覆 $\mathrm{LiFePO}_{4}$ 纳米片的制备及电化学性能研究
}

\author{
王 琦 ${ }^{1}$, 彭大春 ${ }^{1}$ ，马 倩 ${ }^{1}$, 何月德 ${ }^{1,2}$, 刘洪波 ${ }^{1,2}$
}

(湖南大学 1. 材料科学与工程学院; 2. 先进炭材料及应用技术湖南省重点实验室, 长沙 410082)

摘 要: 为改善磷酸铁锂正极材料的倍率性能, 以乙二醇为溶剂, 采用一步溶剂热法制备磷酸铁锂纳米片。再以葡 萄糖为碳前驱体, 对磷酸铁锂纳米片进行炭包覆。通过 $\mathrm{X}$ 射线衍射, $\mathrm{N}_{2}$ 吸脱附曲线、扫描电子显微镜、透射电子显 微镜和循环伏安法等测试方法考察了炭包覆量对磷酸铁锂纳米片结构与电化学性能的影响。结果表明, 制备的磷酸 铁锂为具有较短 $b$ 轴的纳米片状结构, 尺寸约为 $150 \mathrm{~nm} \times 100 \mathrm{~nm} \times 60 \mathrm{~nm}$ 。磷酸铁锂纳米片的倍率性能随炭包覆量的 增加而增强, 当炭包覆量为 $6.4 \mathrm{wt} \%$ 时具有最佳的倍率性能, 在 $0.2 C$ 和 $10 C$ 的电流密度下放电容量分别为 157.3 和 $132.6 \mathrm{mAh} / \mathrm{g}$ 。同时循环稳定性良好, 在 $5 C$ 电流密度下循环 500 次后容量保持率达到了 $80.2 \%$ 。

关 键 词: 磷酸铁锂; 纳米片状结构; 溶剂热; 倍率性能; 循环性能

中图分类号: O646 文献标识码: A

\section{Synthesis and Electrochemical Property of Carbon Coated $\mathrm{LiFePO}_{4} \mathrm{Nanoplates}$}

\author{
WANG Q $i^{1}{ }^{1}$ PENG Da-Chun ${ }^{1}$, MA Qian ${ }^{1}$, HE Yue-De ${ }^{1,2}$, LIU Hong-Bo ${ }^{1,2}$
}

(1. College of Material Science and Engineering, Hunan University, Changsha 410082, China; 2. Hunan Province Key Laboratory for Advanced Carbon Materials Applied Technology, Hunan University, Changsha 410082, China)

\begin{abstract}
To improve the rate performance of $\mathrm{LiFePO}_{4}$ cathode material, $\mathrm{LiFePO}_{4}$ nanoplates were synthesized via one-step glycol-based solvothermal process. Additionally, carbon was employed to coat $\mathrm{LiFePO}_{4}$ with glucose served as carbon source. The morphologies and electrochemical properties of the as-prepared materials were characterized by XRD, BET, SEM, TEM, cyclic voltammetry, etc. Results showed that $\mathrm{LiFePO}_{4}$ nanoplates had the size of approximately $150 \mathrm{~nm} \times 100 \mathrm{~nm} \times 60 \mathrm{~nm}$, displaying a short $b$-axis. The rate performance of $\mathrm{LiFePO}_{4}$ nanoplates was enhanced with the increment of carbon content, and the sample containing $6.4 \mathrm{wt} \%$ carbon (LF0.2) exhibited the most outstanding electrochemical performance, which delivered discharge capacities of $157.3 \mathrm{mAh} / \mathrm{g}$ and $132.6 \mathrm{mAh} / \mathrm{g}$ at $0.2 C$ and $10 C$ rates, respectively. Meanwhile, excellent cycling stability of LF0.2, 80.2\% capacity retention after 500 cycles at $5 C$ rate, was observed.
\end{abstract}

Key words: $\mathrm{LiFePO}_{4}$; nanoplate structure; solvothermal; rate performance; cycling performance

在锂离子电池正极材料中, 磷酸铁锂 $\left(\mathrm{LiFePO}_{4}\right)$ 具有理论比容量高 $(170 \mathrm{mAh} / \mathrm{g})$ 、结构稳定以及绿色无 污染等优点。但较低的电子导电率 $\left(10^{-7} \sim 10^{-10} \mathrm{~S} \cdot \mathrm{cm}^{-1}\right)$ 和锂离子扩散系数 $\left(10^{-12} \sim 10^{-16} \mathrm{~cm}^{2} \cdot \mathrm{S}^{-1}\right)$ 导致其倍率 性能不佳 ${ }^{[1-4]}$, 难以满足迅猛发展的电动汽车锂离
子电池对功率密度的需求 ${ }^{[5-6]}$ 。

改善 $\mathrm{LiFePO}_{4}$ 倍率性能常用方法有表面包覆导 电层 ${ }^{[7-8]}$ 、金属离子掺杂 ${ }^{[9-10]}$ 、控制形貌和减小颗粒 尺寸 ${ }^{[11-13]}$ 等。自 Ceder 等 ${ }^{[14]}$ 根据理论计算提出锂离 子在 $\mathrm{LiFePO}_{4}$ 晶体中沿 $b$ 轴扩散阻力最小以来, 具

收稿日期: 2018-03-19; 收到修改稿日期: 2018-05-30

基金项目：国家自然科学基金(51772083); 湖南省科技重大专项(2018GK1010)

National Natural Science Foundation of China (51772083); Science and Technology Major Projects of Hunan Province (2018GK1010)

作者简介: 王 琦(1994-), 男, 硕士研究生. E-mail: 766245775@qq.com

通讯作者: 刘洪波，教授. E-mail: hndxlhb@163.com 
有较短 $b$ 轴的片状 $\mathrm{LiFePO}_{4}$ 正极材料的可控合成引 起了研究者的广泛关注。Kanamura 等 ${ }^{[15]}$ 在研究水热 反应中 $\mathrm{pH}$ 对 $\mathrm{LiFePO}_{4}$ 形貌的影响时发现, 当前驱体 溶液的 $\mathrm{pH}$ 为 $4<\mathrm{pH}<6.5$ 时, 产物为具有较短 $b$ 轴的 片状结构, 并表现出最佳的电化学性能。Zhang 等 ${ }^{[16]}$ 通过十二烷基苯磺酸钠(SDBS) 辅助水热法制备出 具有较短 $b$ 轴的片状 $\mathrm{LiFePO}_{4}$, 在 $5 C$ 和 $10 C$ 的电流密 度下放电容量分别达 132.3 和 $107.9 \mathrm{mAh} / \mathrm{g}$ 。 Liu 等 ${ }^{[17]}$ 以水和聚乙二醇 400 的二元混合溶剂通过溶剂热法 成功地合成了在 $b$ 轴方向上尺寸约为 $100 \mathrm{~nm}$ 的片状 $\mathrm{LiFePO}_{4}$, 在 $10 \mathrm{C}$ 电流密度下放电容量高达 $137 \mathrm{mAh} / \mathrm{g}$ 。

鉴于乙二醇在溶剂热法合成 $\mathrm{LiFePO}_{4}$ 中具有以 下优点 ${ }^{[18-19]}$ : 一是乙二醇与 $\mathrm{LiFePO}_{4}$ (010)面上的 $\mathrm{Fe}^{2+}$ 有很强的螯合作用, 有利于 $\mathrm{LiFePO}_{4}$ 沿着 $(010)$ 面生长, 形成一定的择优取向; 二是乙二醇的还原 性可以保护 $\mathrm{Fe}^{2+}$ 不被氧化为 $\mathrm{Fe}^{3+}$ 。本研究以乙二醇 为溶剂, 采用一步溶剂热法合成出具有纳米片状结 构的 $\mathrm{LiFePO}_{4}$ 。再以葡萄糖为碳前驱体对 $\mathrm{LiFePO}_{4}$ 纳米片进行炭包覆, 考察了炭包覆量对 $\mathrm{LiFePO}_{4}$ 纳 米片结构与电化学性能的影响。为改善 $\mathrm{LiFePO}_{4}$ 正 极材料的倍率性能、提高锂离子电池的功率密度提 供了理论与实验依据。

\section{1 实验方法}

\section{1 材料的制备}

将 $16 \mathrm{mmol} \mathrm{FeSO}_{4} \cdot 7 \mathrm{H}_{2} \mathrm{O}$ 和 $48 \mathrm{mmol} \mathrm{LiOH} \cdot \mathrm{H}_{2} \mathrm{O}$ 分别加入到 $35 \mathrm{~mL}$ 乙二醇(国药集团化学试剂有限 公司)中, 配制出 $\mathrm{FeSO}_{4}$ 和 $\mathrm{LiOH}$ 的乙二醇溶液。再 将 $16 \mathrm{mmol} \mathrm{H}_{3} \mathrm{PO}_{4}$ 加入到 $10 \mathrm{~mL}$ 乙二醇中, 配制出 $\mathrm{H}_{3} \mathrm{PO}_{4}$ 的乙二醇溶液。

在 $\mathrm{FeSO}_{4}$ 溶液中逐滴加入 $\mathrm{H}_{3} \mathrm{PO}_{4}$ 溶液, 搅拌反 应 $30 \mathrm{~min}$ 后, 将 $\mathrm{LiOH}$ 溶液逐滴加入上述溶液中, 摚拌反应 $30 \mathrm{~min}$ 后将混合溶液转移到 $100 \mathrm{~mL}$ 聚四 氟乙烯反应釜中，放入 $180^{\circ} \mathrm{C}$ 恒温鼓风干燥箱中反 应 $10 \mathrm{~h}$ 。自然冷却后, 分别用去离子水和无水乙醇 将产物中的未反应物质洗涤干净, $80^{\circ} \mathrm{C}$ 下真空干燥 $12 \mathrm{~h}$, 得到浅绿色 $\mathrm{LiFePO}_{4}$ 。

按葡萄糖与 $\mathrm{LiFePO}_{4}$ 分别为 $0: 1 、 0.05: 1$ 、 $0.1: 1$ 和 $0.2: 1$ 的质量配比, 在去离子水中将葡萄 糖与 $\mathrm{LiFePO}_{4}$ 超声分散, 得到悬浮液。在 $80^{\circ} \mathrm{C}$ 下干 燥后, 转移到管式炉中, 在高纯氩气和 $650^{\circ} \mathrm{C}$ 温度下 煓烧 $3 \mathrm{~h}$, 得到不同炭包覆量的 $\mathrm{LiFePO}_{4} / \mathrm{C}$ 正极材料 (按上述葡萄糖和 $\mathrm{LiFePO}_{4}$ 的不同质量配比分别标记
为 LF0、LF0.05、LF0.1 和 LF0.2)。

\section{2 材料的表征}

采用德国布鲁克公司生产的 Bruker D8 型 X 射 线衍射仪(铜靶, $\lambda=0.15406 \mathrm{~nm}$ )对样品进行物相分析, 采用扫描电子显微镜(SEM, Hitachi S-4800 型) 以及 透射电子显微镜(TEM, JEOL-201 1 型)表征样品的 形貌，采用德国耐驰公司生产的同步热分析仪(STA 449 F5 Jupiter)表征样品中的炭含量, 采用美国麦克 公司生产的 Tristar II 3020 型比表面积测试仪表征样 品的比表面积。

\section{3 电池的组装和测试}

以制备的 $\mathrm{LiFePO}_{4} / \mathrm{C}$ 材料为活性物质、乙炔黑 为导电剂、聚偏氟乙烯(PVDF) 为粘结剂, 按照质量 比 $8: 1: 1$ 混合, 采用 $N$-甲基吡咯烷酮(NMP)作为溶 剂配制浆料, 待摚拌均匀后涂覆到铝箔上。在 $80^{\circ} \mathrm{C}$ 鼓风干燥箱中干燥 $4 \mathrm{~h}$, 采用切片机制成 $\phi 14 \mathrm{~mm}$ 的 正极片，并于 $120^{\circ} \mathrm{C}$ 下真空干燥 $24 \mathrm{~h}$ 。

以金属锂片作对电极, $1 \mathrm{~mol} / \mathrm{L} \mathrm{LiPF}_{6} /$ 碳酸乙烯 酯 $(\mathrm{EC})+$ 碳酸二甲酯 $(\mathrm{DMC})+$ 碳酸甲乙酯 $(\mathrm{EMC})$ (体 积比为 $1: 1: 1)$ 为电解液, 在氩气气氛的手套箱中 组装成扣式电池(2016 型)。采用 LAND 电池测试系 统(CT2001A, 武汉蓝电) 对扣式电池进行恒电流充 放电测试, 充放电电压范围为 $2.5 \sim 4.2 \mathrm{~V}$ 。采用电化 学工作站(CHI660C, 上海华辰)对扣式电池进行循 环伏安和交流阻抗测试。

\section{2 结果与讨论}

\subsection{XRD 分析}

图 1 为不同炭包覆量的四个 $\mathrm{LiFePO}_{4} / \mathrm{C}$ 样品的 XRD 图谱。由图可知, 所有样品的衍射峰均对应橄榄 石结构的 $\mathrm{LiFePO}_{4}$ 标准衍射峰位(JCPDS No. 40-1499), 无明显的杂峰出现, 并且所有样品的衍射峰均较尖 锐，且峰强度高，说明经低温溶剂热合成和高温热 处理可以得到结晶良好的 $\mathrm{LiFePO}_{4} / \mathrm{C}$ 样品。图 1 中 没有出现炭的衍射峰, 这是由于样品中炭包覆量较 低(热重结果显示 LF0.05、LF0.1 和 LF0.2 的炭包覆 量分别为 $2.4 \% 、 3.9 \%$ 和 $6.4 \%)$, 其强度较低的 $(002)$ 衍射峰正好被 $\mathrm{LiFePO}_{4}$ 位于 $2 \theta=25.5^{\circ}$ 处较强的(111) 衍射峰掩盖所致。

\subsection{BET 分析}

为了表征 $\mathrm{LiFePO}_{4} / \mathrm{C}$ 样品的比表面积以及孔径 分布, 在液氮温度下对样品进行了氮气吸脱附测 试。如图 2 所示, 所有样品的吸脱附曲线均可归属 于仅存在大孔的III型等温线, 但炭包覆量较大的样 


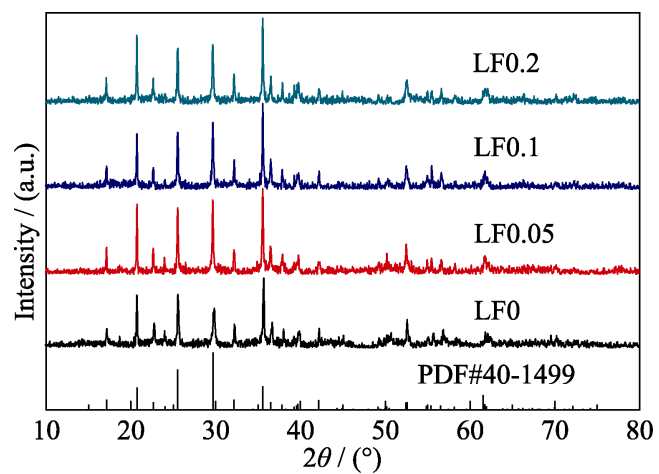

图 $1 \mathrm{LiFePO}_{4} / \mathrm{C}$ 样品的 XRD 图谱

Fig. 1 XRD patterns of $\mathrm{LiFePO}_{4} / \mathrm{C}$ samples

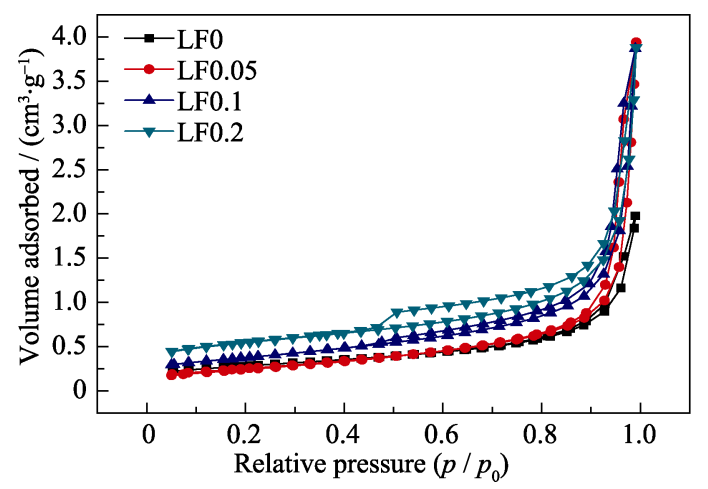

图 $2 \mathrm{LiFePO}_{4} / \mathrm{C}$ 样品的氮气吸脱附曲线

Fig. $2 \mathrm{~N}_{2}$ adsorption-desorption isotherms of $\mathrm{LiFePO}_{4} / \mathrm{C}$ samples

品 LF0.2 在 $p / p_{0}=0.4 \sim 0.9$ 之间出现了明显的滞后环, 说明包覆炭中存在部分介孔。此外, LF0、LF0.05、

LF0.1 和 LF0.2 的 BET 比表面积分别为 15.4、20.0、 29.8 和 $42.8 \mathrm{~m}^{2} / \mathrm{g}$, 其中样品 LF0.2 的比表面积较大, 有利于与电解液充分浸润, 进而提高其电化学性能。

\section{3 形貌观察}

图 3 为不同炭包覆量的四个 $\mathrm{LiFePO}_{4} / \mathrm{C}$ 样品的 扫描电镜照片。可见, 未经炭包覆的 $\mathrm{LiFePO}_{4}$ 纳米 片粒径分布均匀, 约为 $150 \mathrm{~nm} \times 100 \mathrm{~nm} \times 60 \mathrm{~nm}$, 且没有明显的团聚现象(图 3(a)), 说明采用一步溶
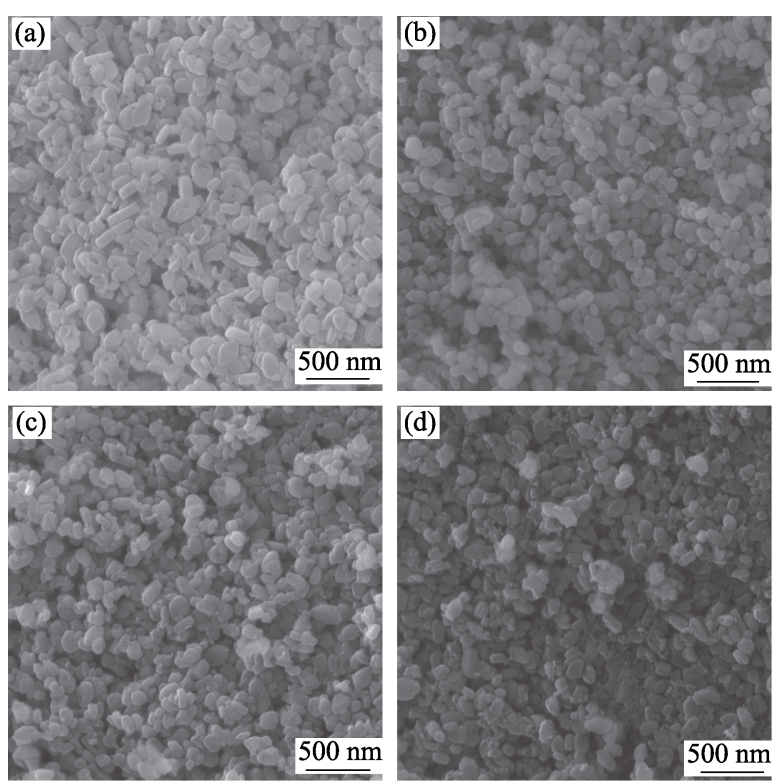

图 3 样品的 SEM 照片

Fig. 3 SEM images of $\mathrm{LiFePO}_{4} / \mathrm{C}$ samples (a) LF0; (b)LF0.05; (c)LF0.1; (d)LF0.2

剂热法可以合成出尺寸均匀且具有较短 $b$ 轴的 $\mathrm{LiFePO}_{4}$ 纳米片。经炭包覆的样品仍保留原 $\mathrm{LiFePO}_{4}$ 纳米片状的形貌，说明炭包覆和热处理对 $\mathrm{LiFePO}_{4}$ 的 纳米片状结构没有太大的影响。但随着炭包覆量的 增加, 炭包覆 $\mathrm{LiFePO}_{4}$ 纳米片表面粗糙度也有所增 大，在炭包覆量较大的样品 LF0.1 和 LF0.2 中还可以 观察到少量散落的炭颗粒, 这可能是由于部分炭未 沉积在 $\mathrm{LiFePO}_{4}$ 表面或从 $\mathrm{LiFePO}_{4}$ 表面脱落所造成。

\section{4 结构与晶形分析}

为了进一步表征 $\mathrm{LiFePO}_{4} / \mathrm{C}$ 样品的形貌以及晶 体取向, 对样品 LF0.2 进行了透射电子显微镜(TEM) 和选区电子衍射(SEAD)测试, 结果如图 4 所示。从 图 4(a)中可观察到样品 LF0.2 呈明显的片状形貌, 且具有较短的 $b$ 轴。图 4(b)为样品 LF0.2 的高分辨 率透射电子显微镜(HRTEM)照片, 如图所示, $\mathrm{LiFePO}_{4}$
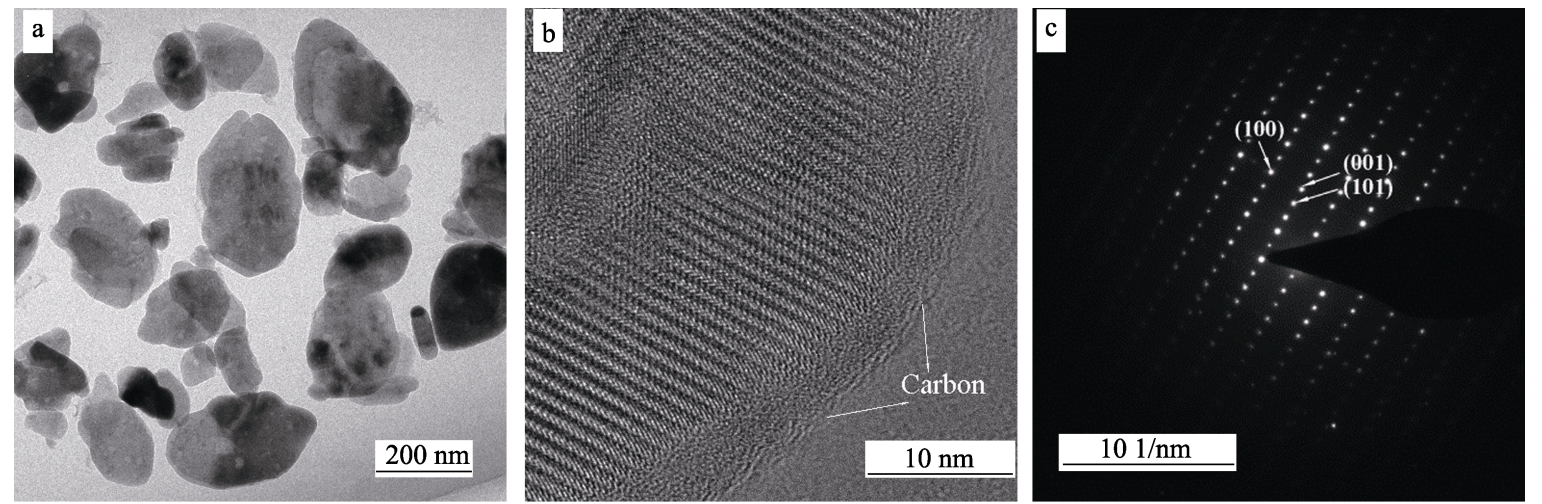

图 4 LF0.2 的 TEM 照片(a)、HRTEM 照片(b)和选区电子衍射照片(c)

Fig. 4 TEM(a), HRTEM(b) and SEAD (c) images of LF0.2 sample 
晶格条纹十分清晰, 说明了样品的结晶性良好, 与 图 1 的 XRD 结果相一致。同时可以观察到 $\mathrm{LiFePO}_{4}$ 表面存在明显且均匀的无定形炭包覆层, 厚度约为 $3 \mathrm{~nm}$ 。图 4(c)为 $\mathrm{LF} 0.2$ 的 SEAD 照片, 可见 $\mathrm{LiFePO}_{4} / \mathrm{C}$ 纳米片为单晶, 且为 $(010)$ 面择优取向。这种片状结 构使 $\mathrm{LiFePO}_{4} / \mathrm{C}$ 样品具有较短的锂离子扩散距离, 有利于提升其倍率性能。

\section{5 电化学性能}

图 5 为不同炭包覆量的四个样品在 $0.1 \mathrm{mV} / \mathrm{s}$ 扫 描速度下的循环伏安曲线, 扫描范围为 $2.5 \sim 4.5 \mathrm{~V}$ 。 从图中可以看出, 所有的曲线均有一对氧化还原峰, 分别对应着 $\mathrm{Li}^{+}$从 $\mathrm{LiFePO}_{4}$ 脱出, 以及 $\mathrm{Li}^{+}$嵌入 $\mathrm{FePO}_{4}$ 的过程 ${ }^{20]}$ 。同时经过仔细比较氧化峰与还原峰之间 的过电位，可以得出 LF0、LF0.05、LF0.1 和 LF0.2 的过电位分别为 205、197、185 和 $174 \mathrm{mV}$ 。上述结 果说明, 随着炭包覆量的增加, 样品的反应可逆性 也在不断增强。

图 6 为不同炭包覆量的四个 $\mathrm{LiFePO}_{4} / \mathrm{C}$ 样品在 不同倍率下的充放电性能。如图 6(a)所示, 未经炭 包覆的样品 LF0 在各倍率下的放电比容量均明显低 于炭包覆样品 LF0.05、LF0.1 和 LF0.2。其中 LF0 在 $0.2 C 、 0.5 C 、 1 C 、 2 C 、 5 C$ 和 $10 C$ 电流密度下的 平均放电比容量分别为 $71.6 、 58.2 、 50.8 、 40.4 、 25.7$
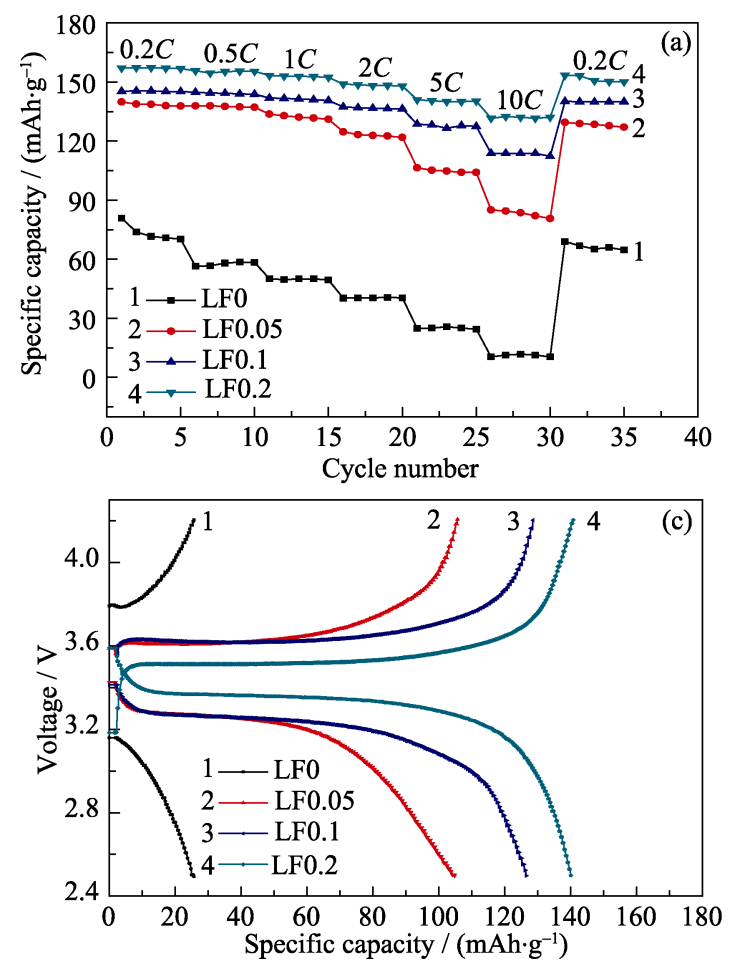

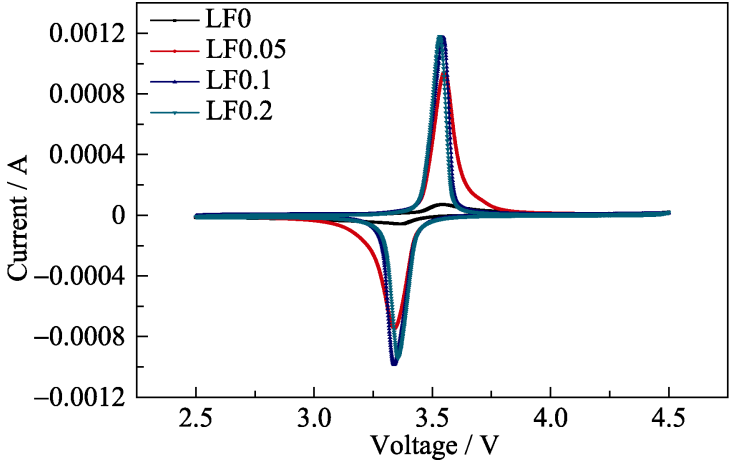

图 $5 \mathrm{LiFePO}_{4} / \mathrm{C}$ 样品的循环伏安曲线

Fig. $5 \mathrm{CV}$ curves of all $\mathrm{LiFePO}_{4} / \mathrm{C}$ samples

和 $11.8 \mathrm{mAh} / \mathrm{g}$, 而三个炭包覆的样品中, 随着炭包 覆量的增加, 倍率性能逐渐提高。其中炭包覆量最 多的样品 LF0.2 在各个倍率下均表现出极佳的电化 学性能, 在 $0.2 C 、 0.5 C 、 1 C 、 2 C 、 5 C$ 和 $10 C$ 的电 流密度下平均放电容量分别为 $157.3 、 155.3 、 152.9$ 、 140.1 和 $132.6 \mathrm{mAh} / \mathrm{g}$ 。即使经过 $10 C$ 电流密度下的充 放电, 在 $0.2 C$ 时的平均放电容量仍达 $152.4 \mathrm{mAh} / \mathrm{g}$, 与未经大电流充放电的结果 $(157.3 \mathrm{mAh} / \mathrm{g})$ 相当。

图 6(b)为不同炭包覆量的四个 $\mathrm{LiFePO}_{4} / \mathrm{C}$ 样品 在 $0.2 C$ 倍率下的第三次充放电曲线, 可见所有样品均 表现出典型的 $\mathrm{LiFePO}_{4}$ 和 $\mathrm{FePO}_{4}$ 之间的两相反应 ${ }^{[21]}$, 但未经炭包覆的样品 LF0 的电化学极化明显大于经
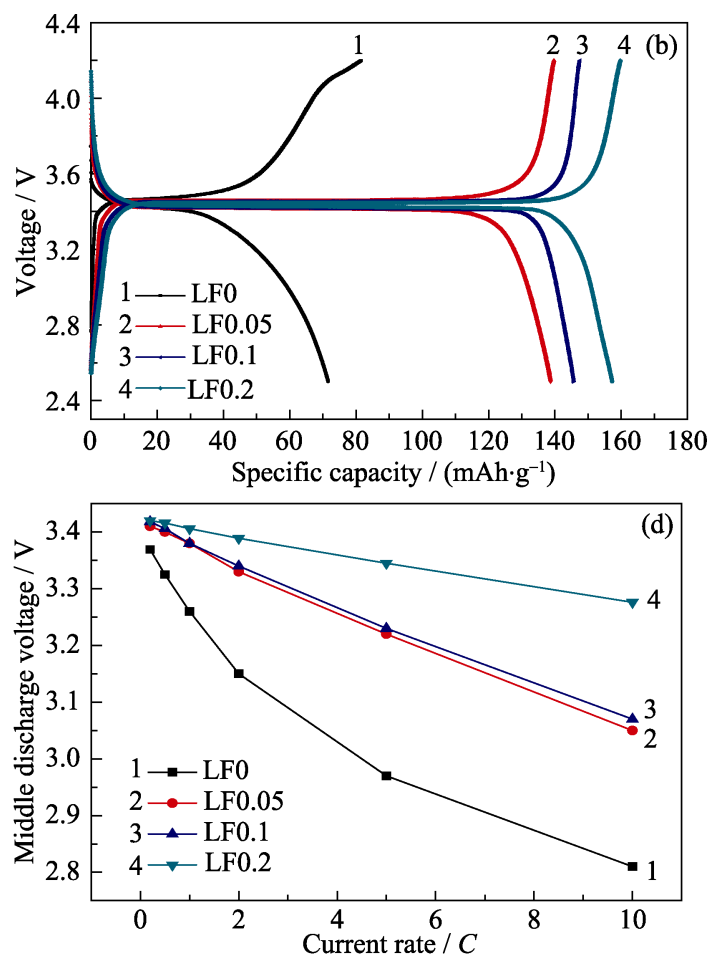

图 6 (a) $\mathrm{LiFePO}_{4} / \mathrm{C}$ 样品的倍率性能; (b) $\mathrm{LiFePO}_{4} / \mathrm{C}$ 样品在 $0.2 \mathrm{C}$ 倍率下的第三次充放电曲线; (c) $\mathrm{LiFePO}_{4} / \mathrm{C}$ 样品在 $5 \mathrm{C}$ 倍率下的第三次充放电曲线; (d)在不同倍率下的中值电压性能图

Fig. 6 (a) Specific capacities of all samples at various rates; Comparison of the third charging and discharging profiles of the all samples at (b) $0.2 C$ rate and (c) $5 C$ rate; (d) The dependency of the middle discharge voltage on current rate in the range of $0.2 C$ to $10 C$ 
炭包覆的样品, 而经炭包覆的三个样品的电化学极 化差值并不大, 说明炭包覆量对 $\mathrm{LiFePO}_{4} / \mathrm{C}$ 样品的 小电流性能影响较小。图 6(c)为样品在 $5 C$ 倍率下的 第三次充放电曲线, 可见经炭包覆的三个样 LF0.05、

LF0.1 和 LF0.2 仍具有明显的电位平台, 且炭包覆量 越大, 电位平台越长, 而 LF0 的电位平台几乎消失, 说明 LF0 在大电流密度下的电化学活性极差。此外, 从不同炭包覆量的三个样品 LF0.05、LF0.1 和 LF0.2 的电化学极化值分别为 $420 、 370$ 和 $160 \mathrm{mV}$, 表明 炭包覆量越多, 样品的大电流性能越佳。

图 6(d)为不同炭包覆量的四个 $\mathrm{LiFePO}_{4} / \mathrm{C}$ 样品 在不同放电密度下的中值电压曲线(放电容量到最 高放电容量一半时的电压)。可见, 未经炭包覆的样 品中值电压在各个电流密度下均小于经炭包覆的样 品, 且炭包覆量最多的 LF0.2 的中值电压随电流密 度增加而衰减的趋势明显小于 LF0.05 和 LF0.1, 说 明炭包覆量较高的样品即使在大电流密度下也能保 持较高的放电电压和功率密度。

图 7 为不同炭包覆量的四个 $\mathrm{LiFePO}_{4} / \mathrm{C}$ 样品在 不同电流密度下的循环性能。由图 7(a)可知, 经炭 包覆的样品 LF0.05、LF0.1 和 LF0.2 在 $1 C$ 电流密度 下经过 100 个循环后容量基本没有衰减, 容量保持 率分别为 $95.4 \% 、 96.5 \%$ 和 $97.2 \%$, 而未经炭包覆的 样品 LF0 的容量保持率仅为 $82.4 \%$, 说明炭包覆可 以明显提高 $\mathrm{LiFePO}_{4}$ 的小电流循环稳定性, 且炭包
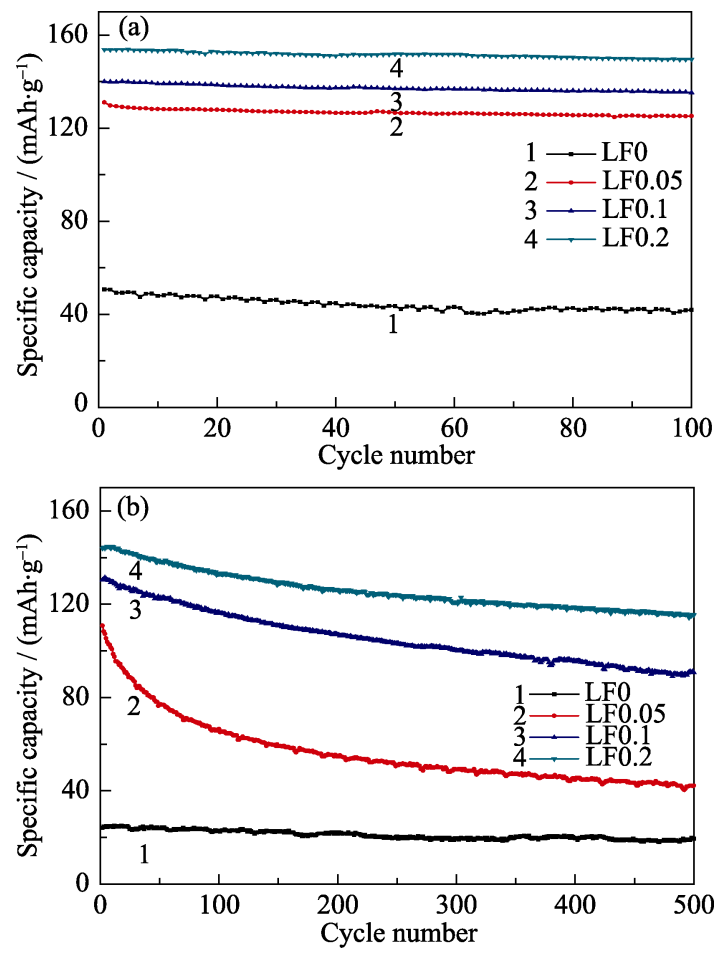

图 $7 \mathrm{LiFePO}_{4} / \mathrm{C}$ 样品的循环性能图

Fig. 7 Cycling performances of $\mathrm{LiFePO}_{4} / \mathrm{C}$ samples (a) $1 C$; (b) $5 C$
覆量对小电流循环稳定性影响较小。图 7(b)为样品 在 $5 C$ 电流密度下的循环性能, 未经炭包覆的 LF0 在 $5 C$ 下的放电容量仅为 $24.6 \mathrm{mAh} / \mathrm{g}$, 其循环稳定性 能不具有可比性。炭包覆量最少的样品 LF0.05 在循 环初期容量下降极快, 500 次循环后容量保持率仅为 $38.2 \%$ 。随着炭包覆量增加, 容量保持率逐渐增加, LF0.1 和 LF0.2 的容量保持率分别为 $69.7 \%$ 和 $80.2 \%$, 说明炭包覆量越大, 样品的大电流循环稳定性越佳。

为了进一步探讨 $\mathrm{LiFePO}_{4} / \mathrm{C}$ 样品电化学行为的 差异, 在样品 $\mathrm{CV}$ 测试完毕后进行了交流阻抗测试, 结果如图 8(a)所示, 所有阻抗图谱由三部分组成, 即高频区半圆与实轴的交点、中高频区的扁圆及低 频区的直线 ${ }^{[22-23]}$ 。根据等效电路拟合得到的各个电 阻值列在表 1 中。由表 1 可知, LF0, LF0.05, LF0.1
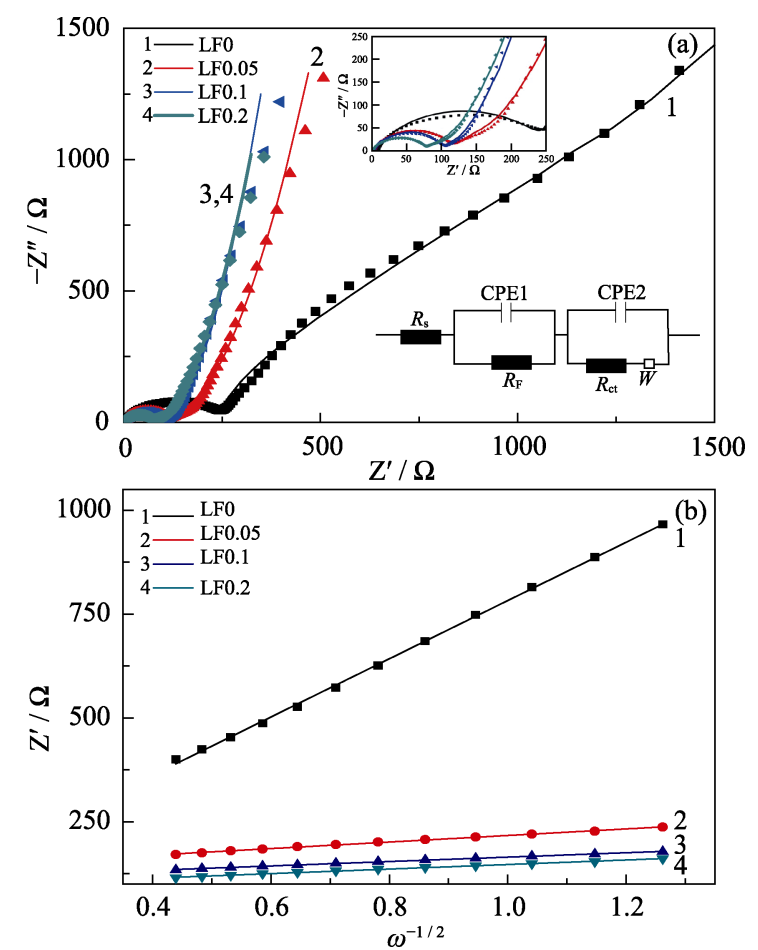

图 8 (a) $\mathrm{LiFePO}_{4} / \mathrm{C}$ 样品的交流阻抗图谱以及对应的等效电 路; (b) $Z^{\prime}-\omega^{-1 / 2}$ 的直线拟合

Fig. 8 (a) The EIS curves and the corresponding equivalent circuit model of $\mathrm{LiFePO}_{4} / \mathrm{C}$ samples; (b) Linear fitting of $Z^{\prime}-\omega^{-1 / 2}$

\section{表 1 等效电路中各个电阻的拟合值以及 对应的锂离子扩散系数}

Table 1 The fitting values of the resistance components in the simplified equivalent circuit and the $\mathrm{Li}^{+}$diffusion coefficients.

\begin{tabular}{ccccc}
\hline Samples & $R_{\mathrm{S}} / \Omega$ & $R_{\mathrm{F}} / \Omega$ & $R_{\mathrm{ct}} / \Omega$ & $D_{\mathrm{Li}}^{+} /\left(\times 10^{-13}, \mathrm{~cm}^{2} \cdot \mathrm{s}^{-1}\right)$ \\
\hline LF0 & 8.1 & 241.8 & 235.2 & 0.067 \\
LF0.05 & 3.8 & 35.7 & 95.7 & 5.300 \\
LF0.1 & 3.5 & 17.6 & 74.1 & 12.000 \\
LF0.2 & 2.2 & 9.3 & 62.9 & 41.500 \\
\hline
\end{tabular}


和 LF0.2 的电荷转移电阻 $R_{\mathrm{ct}}$ 的值分别为 235.2 、95.7、 74.1 和 $62.9 \Omega$, 说明炭包覆可以明显减小电荷转移 阻抗, 并且随着炭包覆量的增加, 电荷转移电阻不 断减小。其中 LF0.2 表现出最小的电荷转移电阻, 这 是因为较大的炭包覆量提高了材料的导电性, 并且 较大的比表面积增加了与电解液的接触。另外根据 公式 $D=R^{2} T^{2} / 2 A^{2} n^{4} F^{4} C^{2} \sigma^{2}$ 计算锂离子扩散系数 ${ }^{[24-26] 。}$ 其中 $R$ 为气体常数, $T$ 为开氏温度, $A$ 为电极面积, $n$ 为每摩尔反应物参与反应时转移的电子数, $F$ 是法拉 第常数, $C$ 为电极材料的锂离子体相浓度, $\sigma$ 为 $Z^{\prime}-\omega^{-1 / 2}$ 直线的拟合斜率，拟合结果如图 8(b)所示。据以上 公式以及拟合结果计算出样品的锂离子扩散系数, 结果如表 1 所示。表明炭包覆可以明显提高样品的 锂离子扩散系数, 随着炭包覆量的增加, 样品的锂 离子扩散系数不断增加。其中 LF0.2 的锂离子扩散 系数达到 $4.15 \times 10^{-12} \mathrm{~cm}^{2} \cdot \mathrm{s}^{-1}$, 远远优于之前报道的 $\mathrm{LiFePO}_{4}$ 颗粒 ${ }^{[24]}$ 。这是由于本研究的 $\mathrm{LiFePO}_{4}$ 形貌 为具有较短 $b$ 轴的片状结构, 大大缩短了锂离子扩 散距离。

\section{3 结论}

采用一步溶剂热法成功制备出具有较短 $b$ 轴的 $\mathrm{LiFePO}_{4}$ 纳米片，纳米片的颗粒尺寸均匀，约为 $150 \mathrm{~nm} \times 100 \mathrm{~nm} \times 60 \mathrm{~nm}$ 。研究了炭包覆量对 $\mathrm{LiFePO}_{4}$ 纳米片正极材料电化学性能的影响, 发现炭包覆可 以明显提高 $\mathrm{LiFePO}_{4}$ 纳米片的电化学性能, 炭包覆 量对 $\mathrm{LiFePO}_{4}$ 大电流性能影响较大。在炭包覆量为 $6.4 \mathrm{wt} \%$ 时, $\mathrm{LiFePO}_{4} / \mathrm{C}$ 具有最佳的电化学性能, 在 $0.2 C 、 0.5 C 、 1 C 、 2 C 、 5 C$ 和 $10 C$ 电流密度下的平 均放电容量分别为 $157.3 、 155.3 、 152.9 、 148.6 、 140.1$ 和 $132.6 \mathrm{mAh} / \mathrm{g}$ 。在 $1 C$ 电流密度下循环 100 次, 容 量几乎无衰减; 在 $5 C$ 电流密度下循环 500 次, 容量 保持率为 $80.2 \%$ 。研究结果表明, 制备的 $\mathrm{LiFePO}_{4} / \mathrm{C}$ 正极材料具有优异的倍率性能和循环性能, 是一种 潜在的同时具有高功率和高能量密度的正极材料。

\section{参考文献:}

[1] PADHI A K, NANJUNDASWAMY K S, GOODENOUGH, et al. Phospho-olivines as positive electrode materials for rechargeable lithium batteries. J. Electrochem. Soc., 1997, 144: 1188-1194.

[2] YUAN L X, WANG Z H, GOODENOUGH, et al. Development and challenges of $\mathrm{LiFePO}_{4}$ cathode material for lithium-ion batteries. Energy Environ. Sci., 2011, 4: 269-284.

[3] ELLIS B L, LEE K T, NAZAR L F. Positive electrode materials for Li-ion and Li-batteries. Chemistry of Materials, 2010, 22(3): 691-714.

[4] WANG C, HONG J. Ionic/electronic conducting characteristics of $\mathrm{LiFePO}_{4}$ cathode materials the determining factors for high rate performance. Electrochemical and Solid-state Letters, 2007, 10(3):
A65-A69.

[5] ZHANG Y, ZHU Y, FU L, et al. Si/C composites as negative electrode for high energy lithium ion batteries. Chinese Journal of Chemistry, 2017, 35(1): 21-29.

[6] CHENG F, LIANG J, TAO Z, et al. Functional materials for rechargeable batteries. Advanced Materials, 2011, 23(15): 1695-1715.

[7] WANG J, SUN X. Understanding and recent development of carbon coating on $\mathrm{LiFePO}_{4}$ cathode materials for lithium-ion batteries. Energy \& Environmental Science, 2012, 5(1): 5163-5185.

[8] NIEN Y H, CAREY J R, CHEN J S. Physical and electrochemical properties of $\mathrm{LiFePO}_{4} / \mathrm{C}$ composite cathode prepared from various polymer-containing precursors. Journal of Power Sources, 2009, 193(2): 822-827.

[9] ZHOU XIN, ZHAO XIN-BING, YU HONG-MING, et al. Electrochemical properties of F-doped $\mathrm{LiFePO}_{4} / \mathrm{C}$ prepared by solid-state synthesis. Journal of Inorganic Materials, 2008, 23(3): 588-590.

[10] WANG D, LI H, SHI S, et al. Improving the rate performance of $\mathrm{LiFePO}_{4}$ by Fe-site doping. Electrochimica Acta, 2005, 50(14): 2955-2958.

[11] NAN C, LU J, LI L, et al. Size and shape control of $\mathrm{LiFePO}_{4}$ nanocrystals for better lithium ion battery cathode materials. Nano Research, 2013, 6(7): 469-477.

[12] MEI R, SONG X, YANG Y, et al. Plate-like $\mathrm{LiFePO}_{4}$ crystallite with preferential growth of (010) lattice plane for high performance Li-ion batteries. RSC Advances, 2014, 4(11): 5746-5752.

[13] WANG F, WANG X, CHANG Z, et al. Electrode materials with tailored facets for electrochemical energy storage. Nanoscale Horizons, 2016, 1(4): 272-289.

[14] MORGAN D, VAN DER VEN A, CEDER G. Li conductivity in $\mathrm{Li}_{x} \mathrm{MPO}_{4}(\mathrm{M}=\mathrm{Mn}, \mathrm{Fe}, \mathrm{Co}, \mathrm{Ni})$ olivine materials. Electrochemical and solid-state letters, 2004, 7(2): A30-A32.

[15] DOKKO K, KOZUMI S, NAKANO H, et al. Particle morphology, crystal orientation, and electrochemical reactivity of $\mathrm{LiFePO}_{4}$ synthesized by the hydrothermal method at $443 \mathrm{~K}$. Journal of Materials Chemistry, 2007, 17(45): 4803-4810.

[16] PEI B, YAO H, ZHANG W, et al. Hydrothermal synthesis of morphology-controlled $\mathrm{LiFePO}_{4}$ cathode material for lithium-ion batteries. Journal of Power Sources, 2012, 220: 317-323.

[17] YANG S, ZHOU X, ZHANG J, et al. Morphology-controlled solvothermal synthesis of $\mathrm{LiFePO}_{4}$ as a cathode material for lithiumion batteries. Journal of Materials Chemistry, 2010, 20(37): 8086-8091.

[18] QIN X, WANG X, XIANG H, et al. Mechanism for hydrothermal synthesis of $\mathrm{LiFePO}_{4}$ platelets as cathode material for lithium-ion batteries. The Journal of Physical Chemistry C, 2010, 114(39): $16806-16812$

[19] WANG B, LIU A, AL ABDULlA W, et al. Desired crystal oriented $\mathrm{LiFePO}_{4}$ nanoplatelets in situ anchored on a graphene cross-linked conductive network for fast lithium storage. Nanoscale, 2015, 7(19): 8819-8828.

[20] LIU W L, TU J P, QIAO Y Q, et al. Optimized performances of core-shell structured $\mathrm{LiFePO}_{4} / \mathrm{C}$ nanocomposite. Journal of Power Sources, 2011, 196(18): 7728-7735.

[21] CHEN R, WU Y, KONG X Y. Monodisperse porous $\mathrm{LiFePO}_{4} / \mathrm{C}$ microspheres derived by microwave-assisted hydrothermal process combined with carbothermal reduction for high power lithium-ion batteries. Journal of Power Sources, 2014, 258: 246-252.

[22] YANG X, MI Y, ZHANG W, et al. Enhanced electrochemical performance of $\mathrm{LiFe}_{06} \mathrm{Mn}_{0.4} \mathrm{PO}_{4} / \mathrm{C}$ cathode material prepared by ferrocene-assisted calcination process. Journal of Power Sources, 2015, 275: 823-830.

[23] ZHOU Y, LU J, DENG C, et al. Nitrogen-doped graphene guided formation of monodisperse microspheres of $\mathrm{LiFePO}_{4}$ nanoplates as the positive electrode material of lithium-ion batteries. Journal of Materials Chemistry A, 2016, 4(31): 12065-12072.

[24] LIU Y, GU J, ZHANG J, et al. Controllable synthesis of nanosized $\mathrm{LiFePO}_{4} / \mathrm{C}$ via a high shear mixer facilitated hydrothermal method for high rate Li-ion batteries. Electrochimica Acta, 2015, 173: $448-457$.

[25] WANG X, HAO H, LIU J, et al. A novel method for preparation of macroporous lithium nickel manganese oxygen as cathode material for lithium ion batteries. Electrochimica Acta, 2011, 56(11): 4065-4069.

[26] TIAN X, ZHOU Y, TU X, et al. Well-dispersed $\mathrm{LiFePO}_{4}$ nanoparticles anchored on a three-dimensional graphene aerogel as high-performance positive electrode materials for lithium-ion batteries. Journal of Power Sources, 2017, 340: 40-50. 